\title{
CLA-producing adjunct cultures improve the nutritional value of sheep cheese fat
}

\author{
E. Renes ${ }^{a}$, P. Gómez-Cortés ${ }^{b}$, M.A. de la Fuente ${ }^{b}$, D.M. Linares ${ }^{c}$, M.E. Tornadijo ${ }^{a}$, J.M. Fresno ${ }^{a, *}$ \\ a Department of Food Hygiene and Technology, Faculty of Veterinary Science, University of León, 24071 León, Spain \\ ${ }^{\mathrm{b}}$ Institute of Food Science Research (CIAL, CSIC-UAM), Madrid, Spain \\ ${ }^{\mathrm{c}}$ Teagasc Food Research Centre, Moorepark, Fermoy, Ireland
}

\section{A R T I C L E I N F O}

\section{Keywords:}

Autochthonous cultures

Cheese

Conjugated linoleic acid

Fatty acid

Sensory characteristics

Sheep

Chemical compounds studied in this article: Linoleic acid (PubChem CID: 5280450)

Linolenic acid (PubChem CID: 5280934)

Rumenic acid (PubChem CID: 5280644)

Vaccenic acid (PubChem CID: 5281127)

\begin{abstract}
A B S T R A C T
The influence of the autochthonous CLA-producing Lactobacillus plantarum TAUL 1588 and Lactobacillus casei subsp. casei SS 1644 strains and the ripening time on the fatty acid (FA) content and sensory characteristics of sheep cheese were investigated. Three cheese types with different cultures and the control cheese were produced in duplicate and ripened for 8 months. 86 individual FA were determined by gas chromatography. Ripening time $(2,90,180$ and 240 days $)$ did not have a significant effect $(P>.05)$ on the FA content. However, the presence of both Lactobacillus CLA-producing strains led to a decrease of the saturated FA content and to 1.30, 1.19 and 1.27 times higher levels of vaccenic acid, CLA and omega-3, respectively, when compared to the control cheese. This combination allowed obtaining sheep milk cheeses with a healthier FA content, without appreciable changes on sensory characteristics. This work could be a promising approach to increase the bioactive fatty acid content of cheeses.
\end{abstract}

\section{Introduction}

The development of foods with beneficial effects on human health is an aspect of great interest to the food industry and especially to the dairy industry (Balthazar et al., 2017). In relation to healthy food trends, milk and fermented dairy products contain lipid compounds that may exert beneficial effects on human health, such as conjugated linoleic acid (CLA) (Koba \& Yanagita, 2014; Lock, Kraft, Rice, \& Bauman, 2009; Tanaka, 2005). In contrast, dairy fat is a poor source in other bioactive lipids as omega-3 fatty acids (Parodi, 2004).

CLA is a group of positional and geometric isomers of octadecadienoic acid which are naturally found in foods derived from ruminants (Shingfield, Bonnet, \& Scollan, 2013). They are mainly biohydrogenation intermediates of dietary polyunsaturated fatty acids (PUFA). Rumenic acid or cis-9, trans-11 $\mathrm{C}_{18: 2}$, the major CLA isomer in dairy foods, is also synthesized in the ruminant mammary gland by the action of the $\Delta-9$ desaturase enzyme on vaccenic acid (trans-11 $\mathrm{C}_{18: 1}$ ) (Bichi et al., 2012), which is another intermediary in the ruminal biohydrogenation.

Balthazar et al. (2017) indicated that the nutritional value of sheep milk, mostly used to cheese manufacturing, could be higher than milk from other ruminants. In addition, various functional dairy products have been developed from sheep milk (Bárcenas et al., 2007). In the
Mediterranean region, most sheep-milk production systems are semiextensive, and grass plays an important role in the flocks' diet. As Gómez-Cortés et al. (2009) pointed out, the supply of grass and thus omega-3 PUFA in the livestock diet represents one of the strategies to enhance the content of these fatty acids and CLA in milk, and subsequently also in cheese (Shingfield et al., 2013).

Several studies have shown that strains of lactic acid bacteria (LAB) are able to synthesize CLA from linoleic acid in culture medium or milk (Gorissen et al., 2010; Ogawa et al., 2005; Renes et al., 2017a). This fact has opened new research lines aimed at increasing the CLA content of cheese by the use of starters or adjunct cultures able to biosynthesize CLA in vitro. The design of autochthonous CLA-producing cultures with application in the production of functional cheeses could increase the diversity of cultures available to the dairy industry, but research in this topic remains still very limited. Taboada, Van Nieuwenhove, Alzogaray, and Medina (2015) have shown that the CLA content of goat cheese could be increased by the use of autochthonous LAB strains and the same was observed by Mohan, Anand, Kalscheur, Hassan, and Hippen (2013) in Cheddar cheese. To the best of our knowledge, there is no information about the effect of the combination of different autochthonous CLA-producing strains used as a mixed adjunct culture on the fatty acid content of sheep milk cheese.

\footnotetext{
* Corresponding author.

E-mail address: jmfreb@unileon.es (J.M. Fresno).
} 
The objective of this study was to investigate the effect of two autochthonous Lactobacillus strains used as adjunct cultures, previously identified and characterized in vitro as CLA producers (Renes et al., 2017a, 2017b), to improve the nutritional value of sheep cheese fat without modifying the sensory properties of the cheeses.

\section{Material and methods}

\subsection{Preparation of cultures}

The autochthonous Lactococcus lactis subsp. lactis TAUL 238 and Lactococcus lactis subsp. cremoris TAUL 1239 strains were selected for their good technological aptitude showed in previous studies (Renes et al., 2017b) and both Lactococcus strains were used as starter cultures. The autochthonous Lactobacillus plantarum TAUL 1588 and Lactobacillus casei subsp. casei SS 1644 strains were selected for their good technological characteristics as well as for their ability to synthesize CLA in skim milk (Renes et al., 2017b). These Lactobacillus strains, individually or in combination, were used as adjunct cultures.

First, each LAB strain was cultured in MRS broth (Oxoid, Hampshire, UK) for Lactobacillus or Elliker broth (BD Difco, New Jersey, USA) for Lactococcus lactis at $30^{\circ} \mathrm{C}$ for $24 \mathrm{~h}$. Then, each strain was cultured in sterilized reconstituted skim milk $(10 \%, w / v)$ at $30^{\circ} \mathrm{C}$ for $24 \mathrm{~h}$. Total viable counts (CFU/g) were determined by plating serial dilutions on MRS or Elliker agar (Oxoid, Hampshire, UK) and no significant differences $(P \geq .05)$ were observed (data not shown). Four autochthonous cultures were designed for cheese-making using the following combination of strains: culture 1 was composed of Lactococcus lactis subsp. lactis TAUL $238(50 \%, v / \mathrm{v})$ and Lactococcus lactis subsp. cremoris TAUL $1239(50 \%, v / v)$; culture 2 was composed of Lactococcus lactis subsp. lactis TAUL $238(30 \%, v / v)$, Lactococcus lactis subsp. cremoris TAUL 1239 (30\%, v/v) and Lactobacillus plantarum TAUL $1588(40 \%, v / v)$; culture 3 was composed of Lactococcus lactis subsp. lactis TAUL $238(30 \%, v / \mathrm{v})$, Lactococcus lactis subsp. cremoris TAUL $1239(30 \%, v / \mathrm{v})$ and Lactobacillus casei subsp. casei SS 1644 $(40 \%, v / \mathrm{v})$ and culture 4 was composed of Lactococcus lactis subsp. lactis TAUL $238(30 \%, v / v)$, Lactococcus lactis subsp. cremoris TAUL 1239 $(30 \%, v / v)$, Lactobacillus plantarum TAUL 1588 (20\%, v/v) and Lactobacillus casei subsp. casei SS 1644 (20\%, v/v). Finally, each autochthonous culture was transferred at $1 \%(v / v)$ to sterilized sheep milk and incubated for $48 \mathrm{~h}$ at $30^{\circ} \mathrm{C}$.

\subsection{Milk and cheese manufacture}

Milk was obtained from a farm of Castellana breed sheep fed ad libitum with natural pasture. Four sheep cheese types were produced in duplicate at pilot scale (Institute of Food Science and Technology (ICTAL), University of León, Spain) according to the following method: milk was pasteurized at $72{ }^{\circ} \mathrm{C}$ for $15 \mathrm{~s}$ and after cooling at $31{ }^{\circ} \mathrm{C}$, calcium chloride $(0.2 \mathrm{~g} / \mathrm{L})$ and starter culture or starter culture plus adjunct $(1 \%, v / v)$ were added. Cheese type 1 was the control and it was produced with the first autochthonous culture (non CLA-producing) indicated in the previous section. Cheese types 2, 3 and 4 were produced with the autochthonous cultures 2, 3, and 4 (CLA-producing), respectively. After 30 min, chymosin (CHY-MAX Extra, 100\% chymosin; 600 IMCU $/ \mathrm{mL}$; Chr. Hansen SL, Madrid, Spain) was added at a rate of $0.05 \mathrm{~mL} / \mathrm{L}$ of milk (diluted in 1:20 with deionized water). After 40-45 min, the curd was cut to rice grain size and the whey was drained off. The curd was transferred to cylindrical moulds $(15 \mathrm{~cm}$ height, $21 \mathrm{~cm}$ diameter) which were pressed for $2 \mathrm{~h}$. Then, cheeses were salted by immersion $\left(18^{\circ}\right.$ Baume, $8^{\circ} \mathrm{C}$ and pH 5.4$)$ for $17 \mathrm{~h}$. Finally, the cheeses were taken to a ripening chamber where they remained at a temperature of $10{ }^{\circ} \mathrm{C}$ and at $80-85 \%$ relative humidity for 240 days.

Samples (each sample corresponded to a whole cheese of $2.5 \mathrm{~kg}$ ) were taken from each cheese type after 2, 90, 180 and 240 days of ripening. Part of the fresh samples was used to carry out the colour, texture and sensory analyses and another portion of the samples was vacuum packed and stored in a freezer $\left(-30^{\circ} \mathrm{C}\right)$ until fatty acid analysis.

\subsection{Determination of the fatty acid content in sheep cheese}

Cheese fat extraction was carried out using n-pentane after grind the sample with a mixture of sand and sodium sulfate (Bodas et al., 2010). Fatty acids were derivatized to methyl esters (FAME) by base-catalyzed methanolysis of glycerides with KOH in methanol (Bichi et al., 2012). FAME were analysed by gas chromatography with two different columns, CP-Sil 88 (100 $\mathrm{m} \times 0.25 \mathrm{~mm}$ i.d., Varian) and SLB-IL111 capillary column $(100 \mathrm{~m} \times 0.25 \mathrm{~mm}$ i.d., Supelco). Detailed gas chromatography methods, identification of unknown FAME and quantification are described in a previous study (de la Fuente, Rodríguez-Pino, \& Juárez, 2015). Sheep milk cheeses were analysed for fatty acid composition after 2, 90, 180 and 240 days of ripening. The health indexes considered were: the omega-6/omega-3 ratio and the atherogenicity index (AI) defined as $\left[\left(\mathrm{C}_{12: 0}+4 \times \mathrm{C}_{14: 0}+\mathrm{C}_{16: 0}\right) /(\Sigma\right.$ unsaturated $\left.\mathrm{FA})\right]$ (Ulbricht \& Southgate, 1991).

\subsection{Texture profile analysis}

Texture analysis was performed on eight cube-shaped $\left(1.9 \mathrm{~cm}^{3}\right)$ samples obtained from each cheese type through the ripening time (2, $90,180,240$ days $)$ at room temperature $\left(20^{\circ} \mathrm{C} \pm 2{ }^{\circ} \mathrm{C}\right)$. The cheese samples were kept at room temperature for approximately $3 \mathrm{~h}$ before analysis and a $0.5 \mathrm{~cm}$ layer from the surface of the cheese was removed. Texture properties of the cheese types were determined in two successive cycles of $80 \%$ compression with a cross-head constant speed of $0.5 \mathrm{~mm} / \mathrm{s}$ using a TZ-XT2 texture analyser (Stable Micro Systems, Godalming, UK). The following textural parameters were determined from the resultant force-time curve using the Texture Expert software (Stable Micro Systems, Godalming, UK): hardness (N), springiness, cohesiveness and chewiness.

\subsection{Colour instrumental measurement}

Colour analyses of sheep milk cheeses were performed throughout the ripening time $(2,90,180,240$ days) using a reflectance colorimeter spectrophotometer CM-700 d (Konica Minolta, Osaka, Japan) provided with a measuring glass head of $8 \mathrm{~mm}$ of diameter, an illuminant D65 and a $10^{\circ}$ observer. The $L^{*}, a^{*}$, and $b^{*}$ colour measurements were determined according to the CIELab colour space, were $\mathrm{L}^{*}$ corresponds to light/dark chromaticity ( $0 \%$ dark to $100 \%$ light), a* to green/red chromaticity ( $-60 \%$ green to $60 \%$ red), and $b^{*}$ to blue/yellow chromaticity ( $-60 \%$ blue to $60 \%$ yellow) (Pinho, Mendes, Alves, \& Ferreira, 2004). The determination by triplicate of colour parameters was carried out measuring 12 different places on the longitudinal cheese sample surface $(1 \mathrm{~cm}$ thick).

\subsection{Sensory analysis}

The sensory evaluation of the 4 cheese types at 2 time points (180 and 240 days of ripening) was carried out by 20 panellists recruited from the Food Hygiene and Technology Department of the University of León, in gender proportion of 12 female and 8 male with ages between 22 and 60 years. Prior to the sensory evaluation of the cheese types, the panel's members were trained in five training sessions of $1 \mathrm{~h}$ with commercial sheep milk cheeses. In this training, odour, flavour and texture attributes were defined and quantified according to the methodology previously described by Bárcenas et al. (2007) and Fresno and Álvarez (2012) for semi-hard and hard cheeses.

Cheese pieces of the same dimension $(4 \mathrm{~cm} \times 1.5 \mathrm{~cm} \times 0.5 \mathrm{~cm})$ of each type were presented to the panel at ambient temperature $\left(20^{\circ} \mathrm{C} \pm 2{ }^{\circ} \mathrm{C}\right)$ and identified with a randomly 3-digit code in one session for cheese types at $180 \mathrm{~d}$ of ripening and another session for 
cheese types at $240 \mathrm{~d}$ of ripening. Panel assessed a total of 15 sensory parameters, divided into 4 main groups: appearance (colour intensity ranging from white to yellowish-brown, holes size and homogenous distribution of the holes), odour (odour intensity, butyric, pungent and mouldy), taste (saltiness, bitterness, sweetness and acidity), and texture (elasticity, adhesiveness, firmness and solubility). These attributes were recorded on a 7-point intensity scale containing the following descriptors: (1) non-existent, (2) very weak, (3) weak, (4) moderate, (5) strong, (6) very strong and (7) extremely strong.

\subsection{Statistical analysis}

Statistical analysis of the experimental data was performed using SPSS v.21 (SPSS, Chicago, IL, USA). The variables studied were tested for the assumption of normality using the Shapiro-Wilk test and for homoscedasticity using the Levene test. Subsequently, a two-way Analysis of Variance (ANOVA) was performed in order to evaluate the effect of the factors culture and ripening time (as fixed factors) and the interaction between them. Tukey's HSD post hoc test was applied at a $5 \%$ significance level in order to compare sheep cheeses produced with different autochthonous cultures throughout the ripening period.

The chromatographic data obtained were also analysed by multivariant statistical techniques (Principal Component Analysis and Euclidean distance squared) by means of the statistical package Minitab 16 (Minitab Incorporated, 2010).

\section{Results and discussion}

\subsection{Cheese fatty acid content}

The study of the fatty acid composition of four sheep cheese types produced with different autochthonous cultures was carried out throughout the ripening (at time points 2, 90, 180 and 240 days). In all the cheese types, no statistically significant differences $(P \geq .05)$ were observed for the 86 fatty acids contents analysed during the different ripening time points. As the ripening time effect and the interaction between cultures and ripening time effects was no significant $(P>.05)$, only means for the cultures effect are presented in Tables 1 , 2 and 3. Similarly to this study, several authors have confirmed that ripening time does not affect the fatty acid content of cheeses (Bodas et al., 2010; dos Santos et al., 2012; Luna, Juárez, \& de la Fuente, 2007).

On the contrary, the type of culture used for sheep cheese-making, had a significant effect $(P<.05)$ on the fatty acid composition. Saturated fatty acid (SFA) contents of the four types are shown in Table 1 . SFA were the predominant fatty acids in the cheeses accounting for $73 \%$ of total FAME in the control cheese type, $72 \%$ in cheese types 2 and 3 , and $70 \%$ in the cheese type 4 . Palmitic acid $\left(C_{16: 0}\right)$ was found in major proportion, followed by myristic acid $\left(\mathrm{C}_{14: 0}\right)$. These results were similar to those described by Bodas et al. (2010) in their study of the fatty acid content of sheep cheeses produced with milk from flocks fed with different diets. From a nutritional point of view, $17 \%$ lower contents of $C_{12: 0}, C_{14: 0}$ and $C_{16: 0}$ in type 4 are important and positive. This is because all these fatty acids are preferentially stored as body fat and are considered as hypercholesterolemic (Parodi, 2004).

Furthermore, one of the most important results of this study was that the combination of two strains of Lactobacillus in the culture used for cheese-making (cheese type 4 ) led to a significant $(P \leq .001)$ higher concentration of $C_{6: 0}, C_{8: 0}$ and $C_{10: 0}(9,17$ and $8 \%$ higher, respectively), which have been associated with positive effects on human health (Nagao \& Yanagita, 2010; Parodi, 2004). These fatty acids are easily digestible, show a low tendency to be stored in the adipose tissue, are preferentially hydrolysed from the triglycerides molecules and are transferred directly from the intestine to the bloodstream. Afterwards, they are transported as free fatty acids to the liver where they are metabolized via mitochondrial $\beta$-oxidation without triglycerides resynthesize, acting as a quick energy source for cells. Furthermore, these
Table 1

Saturated, including odd and branched-chain, fatty acid composition (g/100 g of total fatty acids methyl esters) of the four sheep cheese types produced with different cultures.

\begin{tabular}{|c|c|c|c|c|c|c|}
\hline \multirow[t]{2}{*}{ Variable $^{1}$} & \multicolumn{4}{|c|}{ Cheese types ${ }^{2}$} & \multirow[t]{2}{*}{$\mathrm{SE}^{3}$} & \multirow[t]{2}{*}{ P-value } \\
\hline & 1 & 2 & 3 & 4 & & \\
\hline Total SFA & $73.08^{\mathrm{a}}$ & $71.55^{\mathrm{b}}$ & $71.55^{\mathrm{b}}$ & $70.22^{c}$ & 0.19 & $* * *$ \\
\hline$\Sigma$ Non-BCFA & $71.27^{\mathrm{a}}$ & $69.89^{\mathrm{b}}$ & $69.89^{\mathrm{b}}$ & $68.75^{\mathrm{c}}$ & 0.18 & $* * *$ \\
\hline $4: 0$ & $4.20^{\mathrm{a}}$ & $4.30^{\mathrm{a}}$ & $4.34^{\mathrm{a}}$ & $4.26^{\mathrm{a}}$ & 0.03 & NS \\
\hline $5: 0$ & $0.03^{\mathrm{a}}$ & $0.03^{\mathrm{a}}$ & $0.03^{\mathrm{a}}$ & $0.05^{\mathrm{b}}$ & 0.00 & $* * *$ \\
\hline $6: 0$ & $3.24^{\mathrm{ab}}$ & $3.20^{\mathrm{a}}$ & $3.39^{\mathrm{bc}}$ & $3.54^{\mathrm{c}}$ & 0.03 & $* * *$ \\
\hline $7: 0$ & $0.04^{\mathrm{a}}$ & $0.03^{\mathrm{a}}$ & $0.04^{\mathrm{a}}$ & $0.06^{\mathrm{b}}$ & 0.00 & $* * *$ \\
\hline $8: 0$ & $2.85^{\mathrm{a}}$ & $2.73^{\mathrm{a}}$ & $3.00^{\mathrm{b}}$ & $3.33^{c}$ & 0.04 & $* * *$ \\
\hline 9:0 & $0.06^{\mathrm{a}}$ & $0.05^{\mathrm{b}}$ & $0.06^{\mathrm{a}}$ & $0.10^{c}$ & 0.00 & $* * *$ \\
\hline $10: 0$ & $7.98^{\mathrm{a}}$ & $7.33^{\mathrm{b}}$ & $7.97^{\mathrm{a}}$ & $8.64^{c}$ & 0.09 & $* * *$ \\
\hline $11: 0$ & $0.06^{\mathrm{a}}$ & $0.05^{\mathrm{b}}$ & $0.06^{\mathrm{a}}$ & $0.10^{c}$ & 0.00 & $* * *$ \\
\hline $12: 0$ & $4.39^{\mathrm{a}}$ & $3.89^{\mathrm{b}}$ & $4.12^{\mathrm{c}}$ & $4.11^{\mathrm{c}}$ & 0.04 & $* * *$ \\
\hline $13: 0$ & $0.08^{\mathrm{a}}$ & $0.07^{\mathrm{b}}$ & $0.08^{\mathrm{a}}$ & $0.09^{c}$ & 0.00 & $* * *$ \\
\hline 14:0 & $10.71^{\mathrm{a}}$ & $10.15^{\mathrm{b}}$ & $9.83^{\mathrm{c}}$ & $9.15^{\mathrm{d}}$ & 0.10 & $* * *$ \\
\hline $15: 0$ & $0.98^{\mathrm{a}}$ & $0.82^{\mathrm{b}}$ & $0.85^{\mathrm{b}}$ & $0.88^{c}$ & 0.01 & $* * *$ \\
\hline $16: 0$ & $26.08^{\mathrm{a}}$ & $25.79^{\mathrm{a}}$ & $24.57^{\mathrm{b}}$ & $22.44^{\mathrm{c}}$ & 0.27 & $* * *$ \\
\hline $17: 0$ & $0.63^{\mathrm{a}}$ & $0.55^{\mathrm{b}}$ & $0.55^{\mathrm{b}}$ & $0.49^{c}$ & 0.01 & $* * *$ \\
\hline 18:0 & $9.37^{\mathrm{a}}$ & $10.36^{\mathrm{b}}$ & $10.46^{\mathrm{b}}$ & $10.77^{c}$ & 0.10 & $* * *$ \\
\hline $20: 0$ & $0.26^{\mathrm{a}}$ & $0.29^{\mathrm{b}}$ & $0.27^{\mathrm{a}}$ & $0.24^{c}$ & 0.01 & $* * *$ \\
\hline 21:0 & $0.05^{\mathrm{a}}$ & $0.05^{\mathrm{a}}$ & $0.05^{\mathrm{a}}$ & $0.05^{\mathrm{a}}$ & 0.00 & NS \\
\hline $22: 0$ & $0.11^{\mathrm{a}}$ & $0.10^{\mathrm{ab}}$ & $0.09^{\mathrm{bc}}$ & $0.08^{c}$ & 0.00 & $* *$ \\
\hline 23:0 & $0.06^{\mathrm{a}}$ & $0.05^{\mathrm{ab}}$ & $0.04^{\mathrm{b}}$ & $0.03^{\mathrm{b}}$ & 0.00 & $* *$ \\
\hline $24: 0$ & $0.06^{\mathrm{a}}$ & $0.05^{\mathrm{ab}}$ & $0.05^{\mathrm{ab}}$ & $0.04^{\mathrm{b}}$ & 0.00 & $* *$ \\
\hline$\Sigma$ BCFA & $1.82^{\mathrm{a}}$ & $1.67^{\mathrm{b}}$ & $1.66^{\mathrm{b}}$ & $1.58^{\mathrm{c}}$ & 0.02 & $* * *$ \\
\hline $13: 0$ iso & $0.02^{\mathrm{a}}$ & $0.02^{\mathrm{a}}$ & $0.02^{\mathrm{a}}$ & $0.02^{\mathrm{a}}$ & 0.00 & NS \\
\hline 13:0 anteiso & $0.04^{\mathrm{a}}$ & $0.04^{\mathrm{a}}$ & $0.04^{\mathrm{a}}$ & $0.03^{\mathrm{b}}$ & 0.00 & $*$ \\
\hline $14: 0$ iso & $0.09^{\mathrm{a}}$ & $0.07^{\mathrm{b}}$ & $0.07^{\mathrm{b}}$ & $0.06^{c}$ & 0.00 & $* * *$ \\
\hline $15: 0$ iso & $0.22^{\mathrm{a}}$ & $0.22^{\mathrm{a}}$ & $0.21^{\mathrm{b}}$ & $0.18^{\mathrm{c}}$ & 0.00 & $* * *$ \\
\hline 15:0 anteiso & $0.42^{\mathrm{a}}$ & $0.37^{\mathrm{b}}$ & $0.39^{c}$ & $0.39^{c}$ & 0.00 & $* * *$ \\
\hline $16: 0$ iso & $0.21^{\mathrm{a}}$ & $0.21^{\mathrm{a}}$ & $0.20^{\mathrm{a}}$ & $0.17^{\mathrm{b}}$ & 0.00 & $* * *$ \\
\hline $17: 0$ iso & $0.23^{\mathrm{a}}$ & $0.24^{\mathrm{a}}$ & $0.23^{\mathrm{a}}$ & $0.23^{\mathrm{a}}$ & 0.00 & NS \\
\hline 17:0 anteiso & $0.52^{\mathrm{a}}$ & $0.44^{\mathrm{b}}$ & $0.45^{\mathrm{b}}$ & $0.44^{\mathrm{b}}$ & 0.01 & $* * *$ \\
\hline $18: 0$ iso & $0.06^{\mathrm{a}}$ & $0.06^{\mathrm{a}}$ & $0.06^{\mathrm{a}}$ & $0.05^{\mathrm{b}}$ & 0.00 & $* *$ \\
\hline
\end{tabular}

a-d Means in the same row with different superscripts are significantly different $(P \leq .05)$.

NS $P>.05 ; * P \leq .05 ; * * P \leq .01 ; * * * P \leq .001$.

1 BCFA = branched-chain fatty acids; SFA = saturated fatty acids.

2 1: control cheese made with starters Lactococcus lactis subsp. lactis TAUL 238 and Lc. lactis subsp. cremoris TAUL 1239 strains; 2: cheese made with starters of cheese type 1 and Lactobacillus plantarum TAUL 1588; 3: cheese made with starters of cheese type 1 and Lactobacillus casei subsp. casei SS 1644; 4: cheese made with starters of cheese type 1 and both Lactobacillus strains used in cheese types 3 and 4.

${ }^{3} \mathrm{SE}=$ standard error. Results as mean values of duplicate determination of each fatty acid at $2,90,180$ and 240 days of ripening.

short-chain fatty acids also play an interesting role on the sensory characteristics of cheeses due to their lower perception thresholds in comparison to longer-chain fatty acids (Laskaridis et al., 2013).

Cheese produced with the combination of both Lactobacillus strains in the culture showed the lowest concentration of $\mathrm{C}_{17: 0}$ and branchedchain fatty acids (BCFA) in comparison to cheeses produced with the cultures including one Lactobacillus strain as adjunct culture. This fact showed that the metabolism of the cultures used for cheese-making played an important role in the content of these minor fatty acids in cheese, confirming the relation between the production of odd and $\mathrm{BCFA}$ and the type of microorganism involved in the fermentative process.

The total amount of monounsaturated fatty acids (MUFA) was significantly different $(P<.05)$ increasing from $22.68 \%$ in the cheese type 1 , up to $24.64 \%$ of total fatty acids in cheese type 4 (Table 2). Cheese types 2 and 3 presented an intermediate proportion $(23.96 \%$ and $23.68 \%$, respectively) of MUFA between the other two cheese types. 
Table 2

Monounsaturated fatty acid composition $(\mathrm{g} / 100 \mathrm{~g}$ of total fatty acids methyl esters) of the four sheep cheese types produced with different cultures.

\begin{tabular}{|c|c|c|c|c|c|c|}
\hline \multirow[t]{2}{*}{ Variable $^{1}$} & \multicolumn{4}{|c|}{ Cheese types $^{2}$} & \multirow[t]{2}{*}{$\mathrm{SE}^{3}$} & \multirow[t]{2}{*}{$P$-value } \\
\hline & 1 & 2 & 3 & 4 & & \\
\hline Total MUFA & $22.68^{\mathrm{a}}$ & $23.96^{\mathrm{b}}$ & $23.68^{\mathrm{b}}$ & $24.64^{\mathrm{c}}$ & 0.116 & $* * *$ \\
\hline$\Sigma$ cis-MUFA & $19.79^{\mathrm{a}}$ & $20.93^{\mathrm{b}}$ & $20.63^{b}$ & $20.83^{b}$ & 0.105 & $* * *$ \\
\hline $10: 1$ & $0.26^{\mathrm{a}}$ & $0.26^{\mathrm{a}}$ & $0.26^{\mathrm{a}}$ & $0.26^{\mathrm{a}}$ & 0.003 & NS \\
\hline $12: 1 c-11$ & $0.06^{\mathrm{a}}$ & $0.05^{\mathrm{b}}$ & $0.05^{\mathrm{b}}$ & $0.06^{\mathrm{a}}$ & 0.001 & $* * *$ \\
\hline $14: 1 c-9$ & $0.16^{\mathrm{a}}$ & $0.15^{\mathrm{ab}}$ & $0.14^{\mathrm{b}}$ & $0.12^{\mathrm{c}}$ & 0.004 & $* * *$ \\
\hline$\sum$ cis-16:1 & $0.96^{\mathrm{a}}$ & $0.92^{\mathrm{bc}}$ & $0.91^{\mathrm{c}}$ & $0.94^{\mathrm{ab}}$ & 0.007 & $* * *$ \\
\hline $16: 1 c-7$ & $0.21^{\mathrm{a}}$ & $0.21^{\mathrm{a}}$ & $0.23^{\mathrm{b}}$ & $0.25^{c}$ & 0.004 & $* * *$ \\
\hline $16: 1 c-8$ & $0.02^{\mathrm{a}}$ & $0.02^{\mathrm{a}}$ & $0.02^{\mathrm{a}}$ & $0.03^{\mathrm{b}}$ & 0.002 & $* *$ \\
\hline $16: 1 c-9$ & $0.62^{\mathrm{a}}$ & $0.59^{\mathrm{b}}$ & $0.56^{\mathrm{c}}$ & $0.53^{\mathrm{d}}$ & 0.005 & $* * *$ \\
\hline $16: 1 c-10$ & $0.07^{\mathrm{a}}$ & $0.06^{\mathrm{ab}}$ & $0.06^{\mathrm{ab}}$ & $0.05^{\mathrm{b}}$ & 0.003 & $*$ \\
\hline $16: 1 c-11$ & $0.03^{\mathrm{a}}$ & $0.03^{\mathrm{a}}$ & $0.03^{\mathrm{a}}$ & $0.05^{\mathrm{b}}$ & 0.003 & $* * *$ \\
\hline $16: 1 c-13$ & $0.02^{\mathrm{a}}$ & $0.02^{\mathrm{a}}$ & $0.02^{\mathrm{a}}$ & $0.02^{\mathrm{a}}$ & 0.002 & NS \\
\hline $17: 1 c-9$ & $0.21^{\mathrm{a}}$ & $0.18^{\mathrm{b}}$ & $0.17^{\mathrm{bc}}$ & $0.16^{\mathrm{c}}$ & 0.005 & $* * *$ \\
\hline$\Sigma$ cis-18:1 & $18.11^{\mathrm{a}}$ & $19.31^{\mathrm{b}}$ & $19.05^{\mathrm{b}}$ & $19.23^{\mathrm{b}}$ & 0.102 & $* * *$ \\
\hline $18: 1 c-9$ & $17.00^{\mathrm{a}}$ & $18.14^{\mathrm{b}}$ & $17.81^{\mathrm{b}}$ & $17.88^{\mathrm{b}}$ & 0.100 & $* * *$ \\
\hline $18: 1 c-11$ & $0.65^{\mathrm{a}}$ & $0.67^{\mathrm{a}}$ & $0.69^{\mathrm{a}}$ & $0.76^{\mathrm{b}}$ & 0.014 & $* * *$ \\
\hline $18: 1 c-12$ & $0.30^{\mathrm{a}}$ & $0.33^{\mathrm{b}}$ & $0.33^{\mathrm{b}}$ & $0.32^{\mathrm{b}}$ & 0.003 & $* * *$ \\
\hline $18: 1 c-13$ & $0.03^{\mathrm{a}}$ & $0.03^{\mathrm{a}}$ & $0.04^{\mathrm{b}}$ & $0.05^{c}$ & 0.002 & $* * *$ \\
\hline $18: 1 c-15$ & $0.07^{\mathrm{a}}$ & $0.06^{\mathrm{a}}$ & $0.10^{\mathrm{b}}$ & $0.12^{\mathrm{c}}$ & 0.004 & $* * *$ \\
\hline $18: 1 c-16$ & $0.07^{\mathrm{a}}$ & $0.08^{\mathrm{ab}}$ & $0.09^{b c}$ & $0.10^{c}$ & 0.003 & $* * *$ \\
\hline $20: 1 c-11$ & $0.04^{\mathrm{a}}$ & $0.04^{\mathrm{a}}$ & $0.04^{\mathrm{a}}$ & $0.04^{\mathrm{a}}$ & 0.002 & NS \\
\hline Other $20: 1$ & $0.00^{\mathrm{a}}$ & $0.01^{\mathrm{b}}$ & $0.01^{\mathrm{b}}$ & $0.01^{\mathrm{b}}$ & 0.001 & $* * *$ \\
\hline$\Sigma$ trans-MUFA & $2.89^{\mathrm{a}}$ & $3.04^{\mathrm{b}}$ & $3.05^{b}$ & $3.81^{c}$ & 0.020 & $* * *$ \\
\hline $15: 1$ & $0.05^{\mathrm{a}}$ & $0.04^{\mathrm{b}}$ & $0.04^{\mathrm{b}}$ & $0.04^{\mathrm{b}}$ & 0.002 & $* *$ \\
\hline$\Sigma$ trans-16:1 & $0.22^{\mathrm{a}}$ & $0.21^{\mathrm{a}}$ & $0.22^{\mathrm{a}}$ & $0.24^{\mathrm{b}}$ & 0.005 & $* * *$ \\
\hline $16: 1 t-4$ & $0.01^{\mathrm{a}}$ & $0.01^{\mathrm{a}}$ & $0.01^{\mathrm{a}}$ & $0.01^{\mathrm{a}}$ & 0.001 & NS \\
\hline $16: 1 t-5$ & $0.01^{\mathrm{a}}$ & $0.01^{\mathrm{a}}$ & $0.01^{\mathrm{a}}$ & $0.01^{\mathrm{a}}$ & 0.001 & NS \\
\hline $16: 1 t-6$ & $0.02^{\mathrm{a}}$ & $0.02^{\mathrm{a}}$ & $0.02^{\mathrm{a}}$ & $0.02^{\mathrm{a}}$ & 0.002 & NS \\
\hline $16: 1 t-7+t-8$ & $0.03^{\mathrm{a}}$ & $0.03^{\mathrm{a}}$ & $0.03^{\mathrm{a}}$ & $0.03^{\mathrm{a}}$ & 0.002 & NS \\
\hline $16: 1 t-9$ & $0.12^{\mathrm{a}}$ & $0.12^{\mathrm{a}}$ & $0.12^{\mathrm{a}}$ & $0.14^{\mathrm{b}}$ & 0.002 & $* * *$ \\
\hline $16: 1 t-10$ & $0.02^{\mathrm{a}}$ & $0.03^{\mathrm{ab}}$ & $0.03^{\mathrm{ab}}$ & $0.04^{\mathrm{b}}$ & 0.002 & $* *$ \\
\hline$\Sigma$ trans-18:1 & $2.62^{\mathrm{a}}$ & $2.80^{\mathrm{b}}$ & $2.79^{\mathrm{b}}$ & $3.53^{\mathrm{c}}$ & 0.019 & $* * *$ \\
\hline $18: 1 t-4$ & $0.02^{\mathrm{a}}$ & $0.02^{\mathrm{a}}$ & $0.02^{\mathrm{a}}$ & $0.02^{\mathrm{a}}$ & 0.001 & NS \\
\hline $18: 1 t-5$ & $0.02^{\mathrm{a}}$ & $0.02^{\mathrm{a}}$ & $0.02^{\mathrm{a}}$ & $0.02^{\mathrm{a}}$ & 0.002 & NS \\
\hline $18: 1 t-6+t-7+t-8$ & $0.21^{\mathrm{a}}$ & $0.22^{\mathrm{a}}$ & $0.22^{\mathrm{a}}$ & $0.24^{\mathrm{b}}$ & 0.003 & $* * *$ \\
\hline $18: 1 t-9$ & $0.23^{\mathrm{a}}$ & $0.26^{\mathrm{b}}$ & $0.23^{\mathrm{a}}$ & $0.26^{\mathrm{b}}$ & 0.006 & $* *$ \\
\hline $18: 1 t-10$ & $0.29^{\mathrm{a}}$ & $0.35^{\mathrm{b}}$ & $0.36^{\mathrm{b}}$ & $0.47^{\mathrm{c}}$ & 0.009 & $* * *$ \\
\hline $18: 1 t-11$ (VA) & $1.19^{\mathrm{a}}$ & $1.17^{\mathrm{ab}}$ & $1.13^{\mathrm{b}}$ & $1.55^{\mathrm{c}}$ & 0.013 & $* * *$ \\
\hline $18: 1 t-12$ & $0.41^{\mathrm{a}}$ & $0.46^{\mathrm{b}}$ & $0.46^{\mathrm{b}}$ & $0.54^{c}$ & 0.005 & $* * *$ \\
\hline $18: 1 t-16+c-14$ & $0.26^{\mathrm{a}}$ & $0.31^{\mathrm{b}}$ & $0.36^{\mathrm{c}}$ & $0.44^{\mathrm{d}}$ & 0.003 & $* * *$ \\
\hline
\end{tabular}

a-d Means in the same row with different superscripts are significantly different $(P \leq .05)$.

NS $P>.05 ; * P \leq .05 ; * * P \leq .01 ; * * * P \leq .001$

${ }^{1} \mathrm{AI}=$ atherogenicity index, calculated according to Ulbricht and Southgate (1991); CLA = conjugated linoleic acid; MUFA = monounsaturated fatty acids; PUFA $=$ polyunsaturated fatty acids; RA $=$ rumenic acid; VA $=$ vaccenic acid .

2 1: control cheese made with starters Lactococcus lactis subsp. lactis TAUL 238 and Lc. lactis subsp. cremoris TAUL 1239 strains; 2: cheese made with starters of cheese type 1 and Lactobacillus plantarum TAUL 1588; 3: cheese made with starters of cheese type 1 and Lactobacillus casei subsp. casei SS 1644; 4: cheese made with starters of cheese type 1 and both Lactobacillus strains used in cheese types 3 and 4 .

${ }^{3} \mathrm{SE}=$ standard error. Results as mean values of duplicate determination of each fatty acid at $2,90,180$ and 240 days of ripening.

Oleic acid $\left(\mathrm{C}_{18: 1}\right.$ cis-9) was the fatty acid of the MUFA group that was detected in the highest proportion for the four sheep cheese types, ranging between 78 and $80 \%$ of the total MUFA analysed. Differently, Mohan et al. (2013) detected that Cheddar cheese produced with a CLAproducing strain had a lower content of oleic acid compared to the control cheese. In the present study, it was observed that the use of a combination of cultures including autochthonous CLA-producing strains generated increases of cis- $\mathrm{C}_{18: 1}$ isomers, mainly cis-9 (Table 2). This trend was also observed in the case of the trans-MUFA, being vaccenic acid the major isomer of trans- $\mathrm{C}_{18: 1}$ content in sheep cheese, which represented approximately $44 \%$ of total trans- $\mathrm{C}_{18: 1}$. Similarly,
Table 3

Polyunsaturated fatty acid composition ( $\mathrm{g} / 100 \mathrm{~g}$ of total fatty acids methyl esters) and fatty acid indexes of the four sheep cheese types produced with different cultures.

\begin{tabular}{|c|c|c|c|c|c|c|}
\hline \multirow[t]{2}{*}{ Variable $^{1}$} & \multicolumn{4}{|c|}{ Cheese types ${ }^{2}$} & \multirow[t]{2}{*}{$\mathrm{SE}^{3}$} & \multirow[t]{2}{*}{ P-value } \\
\hline & 1 & 2 & 3 & 4 & & \\
\hline Total PUFA & $4.22^{\mathrm{a}}$ & $4.47^{\mathrm{b}}$ & $4.75^{c}$ & $4.99^{d}$ & 0.036 & $* * *$ \\
\hline$\Sigma$ non-conjugated 18:2 & $2.78^{\mathrm{a}}$ & $3.06^{\mathrm{b}}$ & $3.20^{\mathrm{c}}$ & $3.31^{\mathrm{d}}$ & 0.020 & $* * *$ \\
\hline $18: 2 c-9, t-13+t-8, c-12$ & $0.27^{\mathrm{a}}$ & $0.31^{\mathrm{b}}$ & $0.35^{c}$ & $0.41^{\mathrm{d}}$ & 0.005 & $* * *$ \\
\hline $18: 2 c-9, t-12+t-8, c-13$ & $0.13^{\mathrm{a}}$ & $0.15^{\mathrm{b}}$ & $0.16^{\mathrm{b}}$ & $0.18^{\mathrm{c}}$ & 0.002 & $* * *$ \\
\hline $18: 2 t-9, c-12$ & $0.03^{\mathrm{a}}$ & $0.03^{\mathrm{a}}$ & $0.03^{\mathrm{a}}$ & $0.02^{\mathrm{a}}$ & 0.002 & NS \\
\hline $18: 2 t-11, c-15$ & $0.05^{\mathrm{a}}$ & $0.04^{\mathrm{a}}$ & $0.11^{\mathrm{b}}$ & $0.18^{\mathrm{c}}$ & 0.006 & $* * *$ \\
\hline $18: 2$ other $t, t$ & $0.17^{\mathrm{a}}$ & $0.18^{\mathrm{a}}$ & $0.18^{\mathrm{a}}$ & $0.18^{\mathrm{a}}$ & 0.004 & NS \\
\hline $18: 2 c-9, c-12$ & $2.11^{\mathrm{a}}$ & $2.34^{\mathrm{bc}}$ & $2.37^{\mathrm{b}}$ & $2.32^{\mathrm{c}}$ & 0.013 & $* * *$ \\
\hline $18: 2 c-9, c-15$ & $0.01^{\mathrm{a}}$ & $0.01^{\mathrm{a}}$ & $0.02^{\mathrm{b}}$ & $0.02^{\mathrm{b}}$ & 0.001 & $* * *$ \\
\hline$\Sigma$ conjugated $18: 2$ (CLA) & $0.58^{\mathrm{a}}$ & $0.56^{\mathrm{b}}$ & $0.54^{c}$ & $0.69^{d}$ & 0.004 & $* * *$ \\
\hline $18: 2 t-7, c-9$ & $0.05^{\mathrm{a}}$ & $0.07^{\mathrm{a}}$ & $0.05^{\mathrm{a}}$ & $0.06^{\mathrm{a}}$ & 0.005 & NS \\
\hline $18: 2 c-9, t-11$ (RA) & $0.48^{\mathrm{a}}$ & $0.44^{\mathrm{b}}$ & $0.44^{\mathrm{b}}$ & $0.58^{\mathrm{c}}$ & 0.006 & $* * *$ \\
\hline $18: 2 t-11, c-13$ & $0.01^{\mathrm{a}}$ & $0.01^{\mathrm{a}}$ & $0.01^{\mathrm{a}}$ & $0.01^{\mathrm{a}}$ & 0.001 & NS \\
\hline $18: 2 t-11, t-13$ & $0.01^{\mathrm{a}}$ & $0.01^{\mathrm{a}}$ & $0.01^{\mathrm{a}}$ & $0.01^{\mathrm{a}}$ & 0.001 & NS \\
\hline $18: 2$ other $t, t$ & $0.03^{\mathrm{a}}$ & $0.03^{\mathrm{a}}$ & $0.03^{\mathrm{a}}$ & $0.03^{\mathrm{a}}$ & 0.002 & NS \\
\hline Total 18:2 & $3.36^{\mathrm{a}}$ & $3.62^{\mathrm{b}}$ & $3.74^{c}$ & $3.99^{d}$ & 0.021 & $* * *$ \\
\hline $18: 3 c-9, t-11, c-15$ & $0.05^{\mathrm{a}}$ & $0.06^{\mathrm{a}}$ & $0.06^{\mathrm{a}}$ & $0.08^{\mathrm{b}}$ & 0.004 & $* *$ \\
\hline$\Sigma$ omega-6 & $2.40^{\mathrm{a}}$ & $2.63^{\mathrm{bc}}$ & $2.68^{\mathrm{b}}$ & $2.58^{c}$ & 0.019 & $* * *$ \\
\hline $18: 3 n-6$ & $0.04^{\mathrm{a}}$ & $0.04^{\mathrm{a}}$ & $0.04^{\mathrm{a}}$ & $0.04^{\mathrm{a}}$ & 0.002 & NS \\
\hline $20: 2 n-6$ & $0.03^{\mathrm{a}}$ & $0.02^{\mathrm{a}}$ & $0.02^{\mathrm{a}}$ & $0.02^{\mathrm{a}}$ & 0.002 & NS \\
\hline $20: 3 n-6$ & $0.02^{\mathrm{a}}$ & $0.02^{\mathrm{a}}$ & $0.02^{\mathrm{a}}$ & $0.02^{\mathrm{a}}$ & 0.001 & NS \\
\hline $20: 4 n-6$ & $0.18^{\mathrm{a}}$ & $0.17^{\mathrm{a}}$ & $0.19^{\mathrm{a}}$ & $0.14^{\mathrm{b}}$ & 0.014 & $* *$ \\
\hline $22: 4 n-6$ & $0.03^{\mathrm{a}}$ & $0.03^{\mathrm{a}}$ & $0.04^{\mathrm{a}}$ & $0.03^{\mathrm{a}}$ & 0.003 & NS \\
\hline$\Sigma$ omega-3 & $0.52^{\mathrm{a}}$ & $0.50^{\mathrm{a}}$ & $0.63^{\mathrm{b}}$ & $0.66^{\mathrm{b}}$ & 0.010 & $* * *$ \\
\hline $18: 3 n-3$ & $0.37^{\mathrm{a}}$ & $0.36^{\mathrm{a}}$ & $0.48^{\mathrm{b}}$ & $0.49^{\mathrm{b}}$ & 0.008 & $* * *$ \\
\hline $20: 5 n-3$ & $0.04^{\mathrm{a}}$ & $0.03^{\mathrm{a}}$ & $0.03^{\mathrm{a}}$ & $0.04^{\mathrm{a}}$ & 0.002 & NS \\
\hline $22: 5 n-3$ & $0.09^{\mathrm{a}}$ & $0.09^{\mathrm{a}}$ & $0.10^{\mathrm{a}}$ & $0.12^{\mathrm{b}}$ & 0.005 & $* *$ \\
\hline $22: 6 n-3$ & $0.03^{\mathrm{a}}$ & $0.02^{\mathrm{a}}$ & $0.03^{\mathrm{a}}$ & $0.02^{\mathrm{a}}$ & 0.002 & NS \\
\hline omega-6/omega-3 & $4.63^{\mathrm{a}}$ & $4.23^{\mathrm{b}}$ & $4.24^{\mathrm{b}}$ & $3.89^{c}$ & 0.074 & $* * *$ \\
\hline AI & $2.73^{\mathrm{a}}$ & $2.47^{\mathrm{b}}$ & $2.39^{\mathrm{b}}$ & $2.14^{\mathrm{c}}$ & 0.040 & $* * *$ \\
\hline
\end{tabular}

a-d Means in the same row with different superscripts are significantly different $(P \leq .05)$.

NS P $>0.05 ; * P \leq .05 ; * * P \leq .01 ; * * * P \leq .001$.

${ }^{1} \mathrm{AI}=$ atherogenicity index, calculated according to Ulbricht and Southgate (1991); CLA = conjugated linoleic acid; MUFA = monounsaturated fatty acids; PUFA = polyunsaturated fatty acids; RA = rumenic acid; VA = vaccenic acid.

2 1: control cheese made with starters Lactococcus lactis subsp. lactis TAUL 238 and Lc. lactis subsp. cremoris TAUL 1239 strains; 2: cheese made with starters of cheese type 1 and Lactobacillus plantarum TAUL 1588; 3: cheese made with starters of cheese type 1 and Lactobacillus casei subsp. casei SS 1644; 4: cheese made with starters of cheese type 1 and both Lactobacillus strains used in cheese types 3 and 4 .

${ }^{3} \mathrm{SE}=$ standard error. Results as mean values of duplicate determination of each fatty acid at 2, 90, 180 and 240 days of ripening.

dos Santos et al. (2012) and Taboada et al. (2015) detected 1.49\% $2.01 \%$ of vaccenic acid in goat cheese using CLA-producing strains. High vaccenic acid content in cheese would be desirable since it can be used by humans for the endogenous synthesis of rumenic acid (through the stearoyl Co-A enzyme) providing the beneficial effects on human health that have been attributed to this CLA isomer (Turpeinen et al., 2002).

The type of culture affected the total PUFA content of sheep cheeses in this study (Table 3). Control cheese type presented $4.22 \%$ of total PUFA followed by cheese type 2 with $4.47 \%$. The PUFA proportion of the cheese types 3 and 4 was $4.75 \%$ and $4.99 \%$, respectively. Within this fatty acids group it is important to highlight the great importance of CLA and omega- 3 fatty acids because they have been associated with beneficial effects on human health and the intake of these fatty acids through food is essential to achieve these beneficial effects (Swanson, Block, \& Mousa, 2012; Yang et al., 2015). Cheese type 4 produced using 

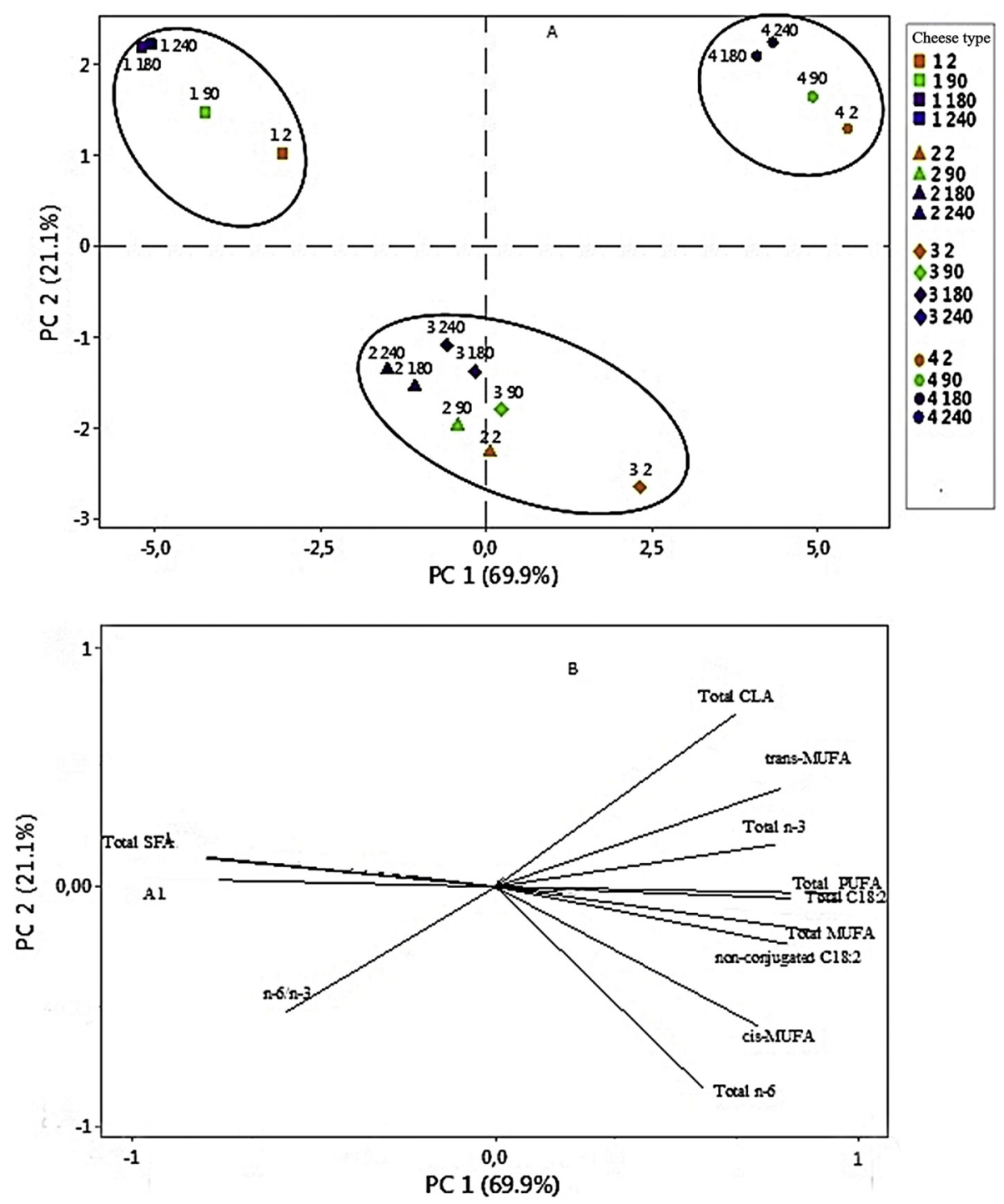

Fig. 1. Score plot (A) and loading vectors (B) of the variables after principal component analysis of the fatty acid composition of the four sheep cheese types throughout ripening (2, 90, 180 and 240 days). CLA = conjugated linoleic acid; MUFA = monounsaturated fatty acids; PUFA = polyunsaturated fatty acids; $\mathrm{BCFA}=$ branched-chain fatty acids; SFA = saturated fatty acids.

the combination of $L b$. plantarum TAUL 1588 and $L b$. casei subsp. casei SS 1644 in the culture contained 19\% more content of total CLA than the control cheese and cheese types 2 and 3. The combination of the two strains could generate more favourable conditions so that linoleate isomerase activity of both strains was optimal. This fact could explain the highest concentration of total CLA observed in cheese type 4 with respect to the control cheese and cheese types 2 and 3 . Gorissen et al. (2011) indicated that linoleate isomerase activity in LAB depends on environmental conditions such as $\mathrm{pH}$ or temperature and it is also strain-dependent. Unfortunately, the mechanisms and factors that affect CLA synthesis by LAB remain unknown and it is necessary to carry out further studies.

The chromatographic method used allowed to detect different CLA isomers. Rumenic acid was the major isomer detected in all cheese types representing $>80 \%$ of total CLA (Table 3). This fact was in accordance with that described by other authors in sheep cheese (Mele et al., 2011; Zlatanos, Laskaridis, Feist, \& Sagredos, 2002). These values were in agreement with those compiled by El-Salam and El-Shibiny (2014), highlighting the great interest of the combination of CLA-producing strains for cheese-making, since it generated higher concentration of this bioactive fatty acid.

$\alpha$-linolenic acid was the major omega-3 fatty acid in all sheep cheeses (Table 3). Cheese types including the Lb. casei subsp. casei SS 1644 strain showed higher concentration of this fatty acid. On the contrary, cheese type 2 containing the $L b$. plantarum TAUL 1588 strain showed a similar $\alpha$-linolenic acid content as the control cheese. Consecuently, $L b$. casei subsp. casei SS1644 strain could be the responsible for the higher $\alpha$-linolenic acid contents in sheep cheeses.

Overall, changes in the fatty acid content of the cheeses gave rise to significant differences $(P \leq .001)$ in the indexes related to human health (Table 3). High omega-6/omega-3 ratio and atherogenicity index (AI) in foods do not imply that the consumption of these foods should be eliminated, but must be kept to a moderate level (Taboada et al., 2015). The omega-6/omega-3 values obtained for control cheese (4.63) were significantly higher $(P \leq .001)$ than those observed in cheese types 2,3 and 4 (4.23, 4.24 and 3.89, respectively). The same trend was observed for the AI values, where the control cheese showed the highest value (2.73) for this index. Cheese types 2 and 3 presented lower $(P \leq .001)$ AI values $(2.47$ and 2.39$)$ than the control cheese. Finally, the cheese type 4 showed the lowest AI values (2.14).

As Astrup et al. (2016) described, current dietary recommendations indicate there is strong evidence that replacing SFA with MUFA and PUFA, reduces plasma LDL-cholesterol and cardiovascular disease risk. These authors also highlighted that according to the report of the 
Table 4

Texture profile analysis and colour measurement of the four cheese types during 240 days of ripening time.

\begin{tabular}{|c|c|c|c|c|c|c|c|c|}
\hline Ripening time & Cheese types $^{1}$ & Hardness (N) & Cohesiveness & Springiness & Chewiness & $\mathrm{L}^{*}$ & $a^{*}$ & $b^{*}$ \\
\hline \multirow[t]{4}{*}{2 days } & 1 & $101.48 \pm 4.76^{\mathrm{aA}}$ & $0.19 \pm 0.02^{\mathrm{aA}}$ & $0.69 \pm 0.03^{\mathrm{aA}}$ & $12.21 \pm 1.02^{\mathrm{aA}}$ & $88.53 \pm 1.67^{\mathrm{aA}}$ & $-1.95 \pm 0.10^{\mathrm{aA}}$ & $14.58 \pm 0.51^{\mathrm{aA}}$ \\
\hline & 2 & $103.34 \pm 6.31^{\mathrm{aA}}$ & $0.18 \pm 0.01^{\mathrm{aA}}$ & $0.69 \pm 0.04^{\mathrm{aA}}$ & $11.59 \pm 0.46^{\mathrm{aA}}$ & $88.92 \pm 1.00^{\mathrm{aA}}$ & $-1.97 \pm 0.12^{\mathrm{aA}}$ & $14.05 \pm 0.46^{\mathrm{aA}}$ \\
\hline & 3 & $166.85 \pm 3.18^{\mathrm{bA}}$ & $0.21 \pm 0.01^{\mathrm{aA}}$ & $0.74 \pm 0.04^{\mathrm{aA}}$ & $25.86 \pm 2.63^{\mathrm{bA}}$ & $89.44 \pm 0.46^{\mathrm{aA}}$ & $-1.90 \pm 0.01^{\mathrm{aA}}$ & $14.64 \pm 0.46^{\mathrm{aA}}$ \\
\hline & 4 & $162.21 \pm 4.37^{\mathrm{bA}}$ & $0.18 \pm 0.02^{\mathrm{aA}}$ & $0.69 \pm 0.03^{\mathrm{aA}}$ & $19.55 \pm 2.76^{\mathrm{cA}}$ & $87.32 \pm 1.82^{\mathrm{aA}}$ & $-1.90 \pm 0.10^{\mathrm{aA}}$ & $14.33 \pm 0.52^{\mathrm{aA}}$ \\
\hline \multirow[t]{4}{*}{90 days } & 1 & $148.44 \pm 1.27^{\mathrm{aB}}$ & $0.13 \pm 0.01^{\mathrm{aB}}$ & $0.53 \pm 0.03^{\mathrm{aB}}$ & $7.65 \pm 5.11^{\mathrm{aAB}}$ & $75.94 \pm 1.27^{\mathrm{aB}}$ & $-2.69 \pm 0.13^{\mathrm{aB}}$ & $18.81 \pm 0.78^{\mathrm{aB}}$ \\
\hline & 2 & $133.45 \pm 8.88^{\mathrm{bB}}$ & $0.13 \pm 0.01^{\mathrm{aB}}$ & $0.36 \pm 0.04^{\mathrm{bB}}$ & $5.98 \pm 0.64^{\mathrm{aC}}$ & $75.75 \pm 2.054^{\mathrm{aB}}$ & $-3.03 \pm 0.11^{\mathrm{bB}}$ & $18.67 \pm 0.42^{\mathrm{aB}}$ \\
\hline & 3 & $180.84 \pm 7.35^{\mathrm{cB}}$ & $0.13 \pm 0.01^{\mathrm{aB}}$ & $0.49 \pm 0.05^{\mathrm{aB}}$ & $12.42 \pm 1.72^{\mathrm{aB}}$ & $77.74 \pm 1.84^{\mathrm{aB}}$ & $-2.25 \pm 0.18^{\mathrm{cB}}$ & $18.78 \pm 0.78^{\mathrm{aB}}$ \\
\hline & 4 & $174.98 \pm 7.43^{\mathrm{cB}}$ & $0.13 \pm 0.02^{\mathrm{aB}}$ & $0.42 \pm 0.04^{\mathrm{abB}}$ & $9.17 \pm 1.20^{\mathrm{aB}}$ & $76.53 \pm 1.47^{\mathrm{aB}}$ & $-2.49 \pm 0.22^{\mathrm{acB}}$ & $21.06 \pm 0.36^{\mathrm{bB}}$ \\
\hline \multirow[t]{4}{*}{180 days } & 1 & $293.42 \pm 7.95^{\mathrm{aC}}$ & $0.14 \pm 0.01^{\mathrm{aB}}$ & $0.38 \pm 0.02^{\mathrm{abC}}$ & $16.11 \pm 1.84^{\mathrm{aC}}$ & $75.75 \pm 2.45^{\mathrm{aB}}$ & $-1.93 \pm 0.26^{\mathrm{aA}}$ & $18.30 \pm 0.77^{\mathrm{aB}}$ \\
\hline & 2 & $230.37 \pm 2.12^{\mathrm{bC}}$ & $0.13 \pm 0.01^{\mathrm{aB}}$ & $0.30 \pm 0.03^{\mathrm{aB}}$ & $8.62 \pm 0.30^{\mathrm{bCB}}$ & $75.12 \pm 2.42^{\mathrm{aB}}$ & $-2.23 \pm 0.21^{\mathrm{aA}}$ & $19.36 \pm 0.88^{\mathrm{aB}}$ \\
\hline & 3 & $265.65 \pm 3.15^{\mathrm{abC}}$ & $0.13 \pm 0.01^{\mathrm{aB}}$ & $0.42 \pm 0.05^{\mathrm{bB}}$ & $13.64 \pm 2.11^{\mathrm{acB}}$ & $75.44 \pm 1.88^{\mathrm{aC}}$ & $-2.25 \pm 0.24^{\mathrm{aB}}$ & $18.59 \pm 0.76^{\mathrm{aB}}$ \\
\hline & 4 & $252.00 \pm 1.73^{\mathrm{abC}}$ & $0.13 \pm 0.01^{\mathrm{aB}}$ & $0.33 \pm 0.05^{\mathrm{aC}}$ & $10.82 \pm 1.35^{\mathrm{bcB}}$ & $74.64 \pm 0.86^{\mathrm{aB}}$ & $-2.29 \pm 0.19^{\mathrm{aB}}$ & $18.00 \pm 0.48^{\mathrm{aB}}$ \\
\hline \multirow[t]{4}{*}{240 days } & 1 & $324.00 \pm 1.52^{\mathrm{aD}}$ & $0.14 \pm 0.01^{\mathrm{aB}}$ & $0.31 \pm 0.03^{\mathrm{aC}}$ & $13.89 \pm 0.93^{\mathrm{aBC}}$ & $75.00 \pm 1.60^{\mathrm{aB}}$ & $-1.89 \pm 0.13^{\mathrm{aA}}$ & $19.43 \pm 0.56^{\mathrm{aB}}$ \\
\hline & 2 & $289.42 \pm 2.81^{\mathrm{abD}}$ & $0.13 \pm 0.01^{\mathrm{aB}}$ & $0.30 \pm 0.03^{\mathrm{aB}}$ & $11.22 \pm 2.10^{\mathrm{aAB}}$ & $74.99 \pm 1.80^{\mathrm{aB}}$ & $-2.22 \pm 0.20^{\mathrm{aA}}$ & $19.48 \pm 0.93^{\mathrm{aB}}$ \\
\hline & 3 & $267.31 \pm 2.09^{\mathrm{bC}}$ & $0.13 \pm 0.01^{\mathrm{aB}}$ & $0.35 \pm 0.03^{\mathrm{aC}}$ & $11.81 \pm 1.82^{\mathrm{aB}}$ & $74.84 \pm 0.86^{\mathrm{aC}}$ & $-2.29 \pm 0.19^{\mathrm{aB}}$ & $19.39 \pm 0.85^{\mathrm{aB}}$ \\
\hline & 4 & $266.03 \pm 2.71^{b D}$ & $0.13 \pm 0.01^{\mathrm{aB}}$ & $0.32 \pm 0.04^{\mathrm{aC}}$ & $12.10 \pm 1.93^{\mathrm{aB}}$ & $74.61 \pm 2.48^{\mathrm{aB}}$ & $-2.22 \pm 0.27^{\mathrm{aAB}}$ & $19.87 \pm 0.54^{\mathrm{aB}}$ \\
\hline
\end{tabular}

a- $\mathrm{d}$ Means \pm standard deviation in the same column with different superscripts (lowercase for differences between batches in the same time of ripening and uppercase for differences between ripening times in each batch) are significantly different $(P \leq .05)$.

1 1: control cheese made with starters Lactococcus lactis subsp. lactis TAUL 238 and Lc. lactis subsp. cremoris TAUL 1239 strains; 2: cheese made with starters of cheese type 1 and Lactobacillus plantarum TAUL 1588; 3: cheese made with starters of cheese type 1 and Lactobacillus casei subsp. casei SS 1644; 4: cheese made with starters of cheese type 1 and both Lactobacillus strains used in cheese types 3 and 4.

A
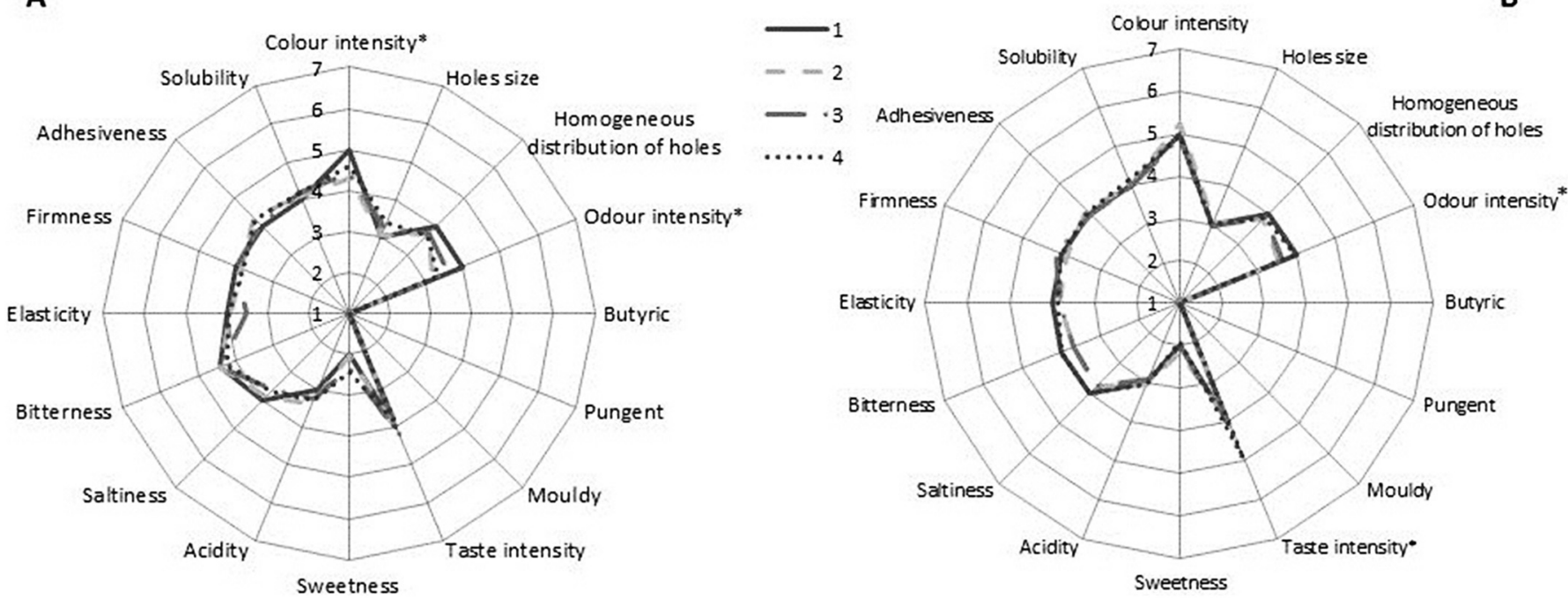

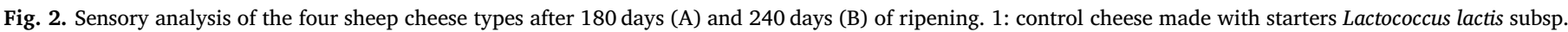

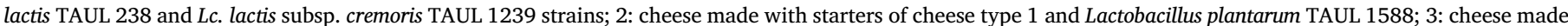

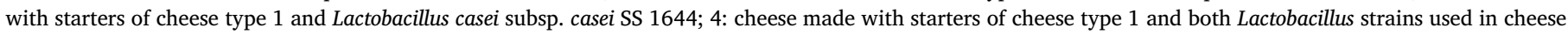
types 3 and 4 . *Denotes attributes significantly different $(P<.05)$ between cheese types.

Dietary Guidelines Advisory Committee (2015), strong and consistent evidence indicates that replacing SFA with PUFA reduces the risk of cardiovascular disease events and coronary mortality. However, this occurred when SFA is replaced by an adequate omega- 6 and omega-3 ratio (approximately 5:1), because replacement with only omega- 6 fatty acids may increase the risk of cardiovascular disease, cancer, and inflammatory and autoimmune diseases, whereas increased levels of omega-3 (a low omega-6/omega-3 ratio) exert suppressive effects (Simopoulos, 2002). Therefore, the results of the present study showed that the use of the autochthonous CLA-producing Lactobacillus plantarum TAUL 1588 and $L b$. casei subsp. casei SS1644 strains as adjunct cultures for cheese-making enhanced the content of the fatty acids with beneficial properties on human health. This effect was higher when both strains were included in the culture (cheese type 4) compared to the culture that did not include CLA-producing strains (control cheese).

Additionally, a principal component analysis (PCA) was performed in order to get a better picture of the fatty acid content showed by the four sheep types throughout ripening (Fig. 1). In the PCA plot, 3 main groups were identified along the first two components, which explained $91 \%$ of the total variation. Cheeses produced with the combination of the two strains were located on the right side of the plot and correlated with cis-, trans-, and total MUFA, non-conjugated and total $\mathrm{C}_{18: 2}$, total CLA, PUFA, omega-6, and omega-3. On the contrary, control cheeses were located on the left side of the plot and associated with the content of total SFA, omega-6/omega-3 ratio and AI. Cheeses that were part of cheese types 2 and 3 constituted a single group that presented intermediate values between cheese types 1 and 4 . Therefore, these results confirm that there is almost no variation related to the cheese-making process itself, and that the type of culture used in cheese-making is the predominant factor influencing cheese fatty acid content (Tables 1, 2 and 3). These results also confirmed that the autochthonous cultures previously detected as CLA-producers in vitro presented this ability when cheeses were produced (Renes et al., 2017b). Therefore, the results of the present study are of great interest in obtaining dairy 
products with improved fatty acid content from sheep milk.

\subsection{Texture, colour and sensory analysis}

In general, texture, colour and sensory data revealed that the use of different adjunct cultures for cheese-making did not lead to important changes on the analysed parameters.

The mean values obtained for the parameters of the texture profile analysis (TPA) and the colour analysis of the four cheese types during ripening are presented in Table 4. Hardness increased up to 240 days of ripening in all the studied cheese types. At this ripening time, cheese types 1 and 2 showed the highest $(P \leq .01)$ hardness values. In general, cohesiveness and chewiness decreased slightly during the first 90 days of ripening, after which remained constant or increased slightly. However, springiness decreased gradually during all the ripening period, reaching after 240 days a final value $50 \%$ lower than the initial at 2 days. At 240 days of ripening, no significant differences $(P \geq .05)$ were observed for cohesiveness, springiness and chewiness between the four cheese types studied. In fact, important changes in texture occur during the first days of cheese ripening because initially the rubbery texture of young cheese is converted to a more homogeneous product and then a gradual change in texture occurs due to hydrolysis of caseins (Pinho et al., 2004).

No significant differences $(P \geq .05)$ were observed for the colour parameters studied between the cheese types throughout 240 days of ripening. In all the cheese types, a decrease in lightness $\left(\mathrm{L}^{*}\right)$ and an increase in redness ( $\left.\mathrm{a}^{*}\right)$ and yellowness $\left(\mathrm{b}^{*}\right)$ during cheese ripening was detected. The changes of the colour parameters of cheese during ripening are strongly related to the physico-chemical changes that take place during cheese ripening because each cheese component reflects specific wavelengths of light. The increase in the a* and $b^{*}$ colour parameters is mainly due to the increase of the dry matter content of the cheeses during ripening (Ávila, Garde, \& Nuñez, 2008).

The mean scores allocated by the panellists for the attributes evaluated in the four cheese types after 180 and 240 days of ripening are shown in Fig. 2. The use of the selected strains as adjunct cultures did not cause any changes $(P \geq .05)$ on the majority of the attributes studied in cheeses after 180 days of ripening. The control cheese showed higher values of colour and odour intensity (Fig. 2A). Regarding the colour attribute, no correlation was found with the results obtained in the instrumental analysis. For this ripening period $\mathrm{L}^{*}, \mathrm{a}^{*}$ and $\mathrm{b}$ * values were similar in the four cheese types studied. None of the cheese types showed anomalous odour. At 240 days of ripening, significant differences were detected $(P<.05)$ for the attributes of odour and flavour intensity (Fig. 2 B). Cheese types 2 and 3 presented the lowest score for odour and taste intensity. No significant correlation was observed between the values obtained for the texture attributes and those resulting from the TPA for neither of the two ripening times. This was observed due to the fact that panellists reported no noteworthy effect of the culture used for cheese-making on the texture attributes of the cheeses. In any cheese type, even for significantly different attributes, the differences were small, always lower than 1 point in the perception scale.

\section{Conclusion}

The combination of the autochthonous Lactobacillus plantarum TAUL 1588 and Lactobacillus casei subsp. casei SS 1644 strains in the culture 4 led to healthier fatty acid content in the sheep cheese type produced, in comparison to the control cheese. The sheep cheese produced with the combination of the two Lactobacillus strains as adjunct cultures showed higher vaccenic acid, CLA and omega-3 levels, as well as lower $\mathrm{C}_{\text {14:0 }}$ and $\mathrm{C}_{16: 0}$ content, omega-6/omega- 3 ratio and $\mathrm{AI}$ than the control cheese. In addition, these fatty acid content changes in sheep cheeses did not generate differences on their sensory characteristics. Overall, the use of these autochthonous CLA-producing cultures could be a promising approach to improve the nutritional quality of cheese fat with special emphasis on bioactive fatty acids, which would be of special interest to the dairy industry in order to meet consumer demands.

\section{Acknowledgments}

The authors are grateful to the University of León (León, Spain) for granting a $\mathrm{PhD}$ fellowship to Erica Renes Bañuelos. Pilar Gómez-Cortés was subsidized with a Juan de la Cierva research contract from the Ministerio de Economía y Competitividad. The authors also wish to acknowledge the Consortium for Ovine Promotion (Villalpando, Zamora, Castilla-León, Spain). The authors thank to F.J. Zorita for his technical assistance.

\section{References}

Astrup, A., Rice Bradley, B., Brenna, J., Delplanque, B., Ferry, M., \& Torres-Gonzalez, M. (2016). Regular-fat dairy and human health: A synopsis of Symposia presented in Europe and North America (2014-2015). Nutrients, 8(8), 463.

Ávila, M., Garde, S., \& Nuñez, M. (2008). The influence of some manufacturing and ripening parameters on the colour of ewes' milk cheese. Milchwissenschaft, 63(2), $160-164$.

Balthazar, C. F., Pimentel, T. C., Ferrão, L. L., Almada, C. N., Santillo, A., Albenzio, M., Cruz, A. G. (2017). Sheep Milk: Physicochemical characteristics and relevance for functional food development. Comprehensive Reviews in Food Science and Food Safety, 16(2), 247-262.

Bárcenas, P., Pérez Elortondo, F. J., Albisu, M., Mège, J., Bivar Roseiro, L., Francesca Scintu, M., ... Lavanchy, P. (2007). An international ring trial for the sensory evaluation of raw ewes' milk cheese texture. International Dairy Journal, 17(9), 1139-1147.

Bichi, E., Toral, P. G., Hervás, G., Frutos, P., Gómez-Cortés, P., Juárez, M., \& de la Fuente, M. A. (2012). Inhibition of $\Delta 9$-desaturase activity with sterculic acid: Effect on the endogenous synthesis of cis-9 18:1 and cis-9, trans-11 18:2 in dairy sheep. Journal of Dairy Science, 95(9), 5242-5252.

Bodas, R., Manso, T., Mantecón, A. R., Juárez, M., De la Fuente, M. A. \& Gómez-Cortés, P. (2010). Comparison of the fatty acid profiles in cheeses from ewes fed diets supplemented with different plant oils. Journal of Agricultural and Food Chemistry, 58(19), 10493-10502.

dos Santos, K. M. O., Bomfim, M. A. D., Vieira, A. D. S., Benevides, S. D., Saad, S. M. I., Buriti, F. C. A., \& Egito, A. S. (2012). Probiotic caprine Coalho cheese naturally enriched in conjugated linoleic acid as a vehicle for Lactobacillus acidophilus and beneficial fatty acids. International Dairy Journal, 24(2), 107-112.

El-Salam, M. H. A., \& El-Shibiny, S. (2014). Conjugated linoleic acid and vaccenic acid contents in cheeses: An overview from the literature. Journal of Food Composition and Analysis, 33(1), 117-126.

Fresno, M., \& Álvarez, S. (2012). Chemical, textural and sensorial changes during the ripening of Majorero goat cheese. International Journal of Dairy Technology, 65(3), 393-400.

Gómez-Cortés, P., Frutos, P., Mantecón, A. R., Juárez, M., de la Fuente, M. A., \& Hervás, G. (2009). Effect of supplementation of grazing dairy ewes with a cereal concentrate on animal performance and milk fatty acid profile. Journal of Dairy Science, 92(8), 3964-3972.

Gorissen, L., Raes, K., Weckx, S., Dannenberger, D., Leroy, F., De Vuyst, L., \& De Smet, S. (2010). Production of conjugated linoleic acid and conjugated linolenic acid isomers by Bifidobacterium species. Applied Microbiology and Biotechnology, 87(6), 2257-2266.

Gorissen, L., Weckx, S., Vlaeminck, B., Raes, K., De Vuyst, L., De Smet, S., \& Leroy, F. (2011). Linoleate isomerase activity occurs in lactic acid bacteria strains and is af fected by pH and temperature. Journal of Applied Microbiology, 111(3), 593-606.

Koba, K., \& Yanagita, T. (2014). Health benefits of conjugated linoleic acid (CLA). Obesity Research \& Clinical Practice, 8(6), 525-532.

de la Fuente, M. A., Rodríguez-Pino, V., \& Juárez, M. (2015). Use of an extremely polar $100-\mathrm{m}$ column in combination with a cyanoalkyl polysiloxane column to complemen the study of milk fats with different fatty acid profiles. International Dairy Journal, 47, $52-63$.

Laskaridis, K., Serafeimidou, A., Zlatanos, S., Gylou, E., Kontorepanidou, E., \& Sagredos, A. (2013). Changes in fatty acid profile of feta cheese including conjugated linoleic acid. Journal of the Science of Food and Agriculture, 93(9), 2130-2136.

Lock, A. L., Kraft, J., Rice, B. H., \& Bauman, D. E. (2009). Biosynthesis and biological activity of rumenic acid: A natural CLA isomer. In F. Destaillats, J. L. Sébédio, F. Dionisi, \& J. M. Chardigny (Eds.). Trans fatty acids in human nutrition (pp. 195-230). (2nd ed.). Bridgwater: The Oily Press.

Luna, P., Juárez, M., \& de la Fuente, M. A. (2007). Conjugated linoleic acid content and isomer distribution during ripening in three varieties of cheeses protected with designation of origin. Food Chemistry, 103(4), 1465-1472.

Mele, M., Contarini, G., Cercaci, L., Serra, A., Buccioni, A., Povolo, M., ... Secchiari, P. (2011). Enrichment of Pecorino cheese with conjugated linoleic acid by feeding dairy ewes with extruded linseed: Effect on fatty acid and triglycerides composition and on oxidative stability. International Dairy Journal, 21(5), 365-372.

Mohan, M. S., Anand, S., Kalscheur, K. F., Hassan, A. N., \& Hippen, A. R. (2013). Starter cultures and cattle feed manipulation enhance conjugated linoleic acid concentrations in Cheddar cheese. Journal of Dairy Science, 96(4), 2081-2094.

Nagao, K., \& Yanagita, T. (2010). Medium-chain fatty acids: Functional lipids for the 
prevention and treatment of the metabolic syndrome. Pharmacological Research, 61(3), 208-212.

Ogawa, J., Kishino, S., Ando, A., Sugimoto, S., Mihara, K., \& Shimizu, S. (2005)

Production of conjugated fatty acids by lactic acid bacteria. Journal of Bioscience and Bioengineering, 100(4), 355-364.

Parodi, P. W. (2004). Milk fat in human nutrition. Australian Journal of Dairy Technology, $59(1), 3$.

Pinho, O., Mendes, E., Alves, M. M., \& Ferreira, I. M. P. L. V. O. (2004). Chemical, physical, and sensorial characteristics of "Terrincho" ewe cheese: Changes during ripening and intravarietal comparison. Journal of Dairy Science, 87(2), 249-257.

Renes, E., Linares, D. M., González, L., Fresno, J. M., Tornadijo, M. E., \& Stanton, C. (2017a). Production of conjugated linoleic acid and gamma-aminobutyric acid by autochthonous lactic acid bacteria and detection of the genes involved. Journal of Functional Foods, 34, 340-346.

Renes, E., Linares, D. M., González, L., Fresno, J. M., Tornadijo, M. E., \& Stanton, C. (2017b). Study of the conjugated linoleic acid synthesis by Lactobacillus strains an by different co-cultures designed for this ability. Journal of Functional Foods, 35, 74-80.

Shingfield, K. J., Bonnet, M., \& Scollan, N. D. (2013). Recent developments in altering the fatty acid composition of ruminant-derived foods. Animal, 7(1), 132-162.

Simopoulos, A. P. (2002). The importance of the ratio of omega-6/omega-3 essential fatty acids. Biomedicine \& Pharmacotherapy, 56(8), 365-379.

Swanson, D., Block, R., \& Mousa, S. A. (2012). Omega-3 fatty acids EPA and DHA: Health benefits throughout life. Advances in Nutrition: An International Review Journal, $3(1), 1-7$.

Taboada, N., Van Nieuwenhove, C., Alzogaray, S. L., \& Medina, R. (2015). Influence of autochthonous cultures on fatty acid composition, esterase activity and sensory profile of Argentinean goat cheeses. Journal of Food Composition and Analysis, 40, 86-94.

Tanaka, K. (2005). Occurrence of conjugated linoleic acid in ruminant products and its physiological functions. Animal Science Journal, 76(4), 291-303.

Turpeinen, A. M., Mutanen, M., Aro, A., Salminen, I., Basu, S., Palmquist, D. L., \& Griinari, J. M. (2002). Bioconversion of vaccenic acid to conjugated linoleic acid in humans. The American Journal of Clinical Nutrition, 76(3), 504-510.

Ulbricht, T. L. V., \& Southgate, D. A. T. (1991). Coronary heart disease: Seven dietary factors. The Lancet, 338(8773), 985-992.

Yang, B., Chen, H., Stanton, C., Ross, R. P., Zhang, H., Chen, Y. Q., \& Chen, W. (2015). Review of the roles of conjugated linoleic acid in health and disease. Journal of Functional Foods, 15, 314-325.

Zlatanos, S., Laskaridis, K., Feist, C., \& Sagredos, A. (2002). CLA content and fatty acid composition of Greek Feta and hard cheeses. Food Chemistry, 78(4), 471-477. 